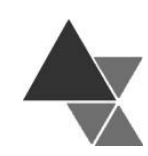

\title{
Capacidade instalada para o fortalecimento do Sistema de Segurança Alimentar e Nutricional em Sergipe
}

\author{
Tatiana Canuto Silva ${ }^{1}$, Andhressa Fagundes² e Danielle Góes da Silva ${ }^{3}$
}

O artigo apresenta um estudo transversal com o objetivo de avaliar o potencial para o fortalecimento do Sistema de Segurança Alimentar e Nutricional (SISAN) em cinco municípios do estado de Sergipe, por meio de coleta de dados direta e pesquisa documental. Foram utilizados dois instrumentos para coleta de dados: check list sobre o Sistema Nacional de Segurança Alimentar e Nutricional, proposto pela gestão nacional, e uma matriz de indicadores de Segurança Alimentar e Nutricional (SAN), proposta pela Universidade Federal da Bahia, ambos adaptados à realidade do estado de Sergipe. Os resultados mostram o risco de Insegurança Alimentar em todos os municípios estudados, reforçando a necessidade do Sistema. A partir dos resultados é possível identificar a frágil capacidade instalada para o Sistema nos municípios que já o tem implantado; a necessidade de maior interesse dos gestores pela adesão ao sistema, nos territórios que ainda não o aderiram, e pelas ações de SAN em suas respectivas localidades; necessidade de fortalecer a participação da sociedade civil na luta para a efetivação destas políticas. Ressalta-se a relevância de fortalecer o SISAN nos municípios que já o aderiram, e implantar naqueles que ainda não aderiram, considerando que será exequível o monitoramento das ações e programas de SAN.

Palavras-chave: segurança alimentar, indicadores, nutrição, municípios.

\section{Installed capacity for the System's Strengthening of Food and Nutritional Security in Sergipe}

Transversal study in order to evaluate the capacity to strengthen the Food Security and Nutritional System in five cities of the state of Sergipe. Two instruments for data collection were used: check list on the National Food Security and Nutritional System, proposed by the national administration, and Food Security and Nutrition indicators, proposed by the Federal University of Bahia, both adapted to the reality of the state of Sergipe. The results show the risk of Food Insecurity in all the studied municipalities, reinforcing the need of the System. From the results it is possible to identify the fragile capacity installed for the System in the municipalities that have already implemented it; the need of greater interest of the managers by the adhesion to the system, in the territories that have not yet adhered to it, and by the actions of SANs in their respective localities; need to strengthen the participation of civil society in the struggle for the implementation of these policies. The importance of

\footnotetext{
${ }^{1}$ Mestranda em Ciências da Nutrição pela Universidade Federal de Sergipe (UFS). Pós-Graduanda em Segurança Alimentar e Nutricional (Unesp). Endereço para correspondência: Universidade Federal de Sergipe - Av. Marechal Rondon, s/n, Bairro Rosa Elze, São Cristovão - SE, CEP 49.100-000. Telefone: (079) 991207737. E-mail: tatiicanuto@hotmail.com 2 Docente Adjunta da Universidade Federal de Sergipe (UFS).

${ }^{3}$ Docente Adjunta da Universidade Federal de Sergipe (UFS).
} 
strengthening SISAN in the municipalities that have already adhered to it, as well as in those that have not yet adhered to, should be highlighted, considering that monitoring of SAN actions and programs will be feasible.

Keywords: food security, indicators, nutrition, municipalities.

\section{INTRODUÇÃO}

A definição de Segurança Alimentar e Nutricional (SAN) encontra-se em constante composição, visto que a questão alimentar está ligada a diversos setores, embates e interesses. É um conceito que progride à medida que ocorrem mudanças na organização social[1], como também quando recebe intervenção direta em resposta às necessidades populacionais ${ }^{[2]}$.

Segundo a Lei Orgânica de Segurança Alimentar e Nutricional (LOSAN), instituída em 2006, define-se como SAN a efetivação do direito ao acesso frequente e perdurável aos alimentos de qualidade, em quantidade suficiente, de forma sustentável sem prejudicar outras necessidades primordiais. Essa lei afirma, ainda, que a alimentação adequada é um direito substancial, inerente à dignidade do homem ${ }^{[3]}$.

A partir da LOSAN foi criado o Sistema Nacional de Segurança Alimentar e Nutricional (SISAN), devido à necessidade de avaliação e realinhamento periódico das políticas públicas do país, para que os objetivos das políticas fossem alcançados. O SISAN é um sistema público que tem como objetivo assegurar o Direito Humano à Alimentação Adequada (DHAA) ${ }^{[4]}$, permite a gestão intersetorial participativa e a articulação de forma complementar entre as esferas do governo, para a efetivação das políticas promotoras de SAN[5], sendo recomendado pelo Governo Federal a implantação do SISAN, em todos os municípios.

São integrantes do SISAN e, portanto, requisitos mínimos para a sua implantação: os Conselhos de Segurança Alimentar e Nutricional (CONSEA), a Câmara Interministerial ou Intersetorial de Segurança Alimentar e Nutricional (CAISAN), órgãos e entidades de SAN da União, dos Estados, do Distrito Federal e dos Municípios e as instituições privadas, elaboração do plano municipal de $\mathrm{SAN}$, bem como a realização de Conferências de Segurança Alimentar e Nutricional[ $[$.
Vários progressos foram alcançados no campo da SAN nos últimos anos, especialmente no que se refere à melhoria do acesso dos brasileiros aos alimentos, em decorrência de uma série de ações e programas focados no enfrentamento da fome e da pobrezal] como o Programa Bolsa Familia, o Programa Nacional de Alimentação Escolar (PNAE), a compra mínima de 30\% diretamente da agricultura familiar para o PNAE, dentre outros ${ }^{[8]}$. O país tem alcançado, dessa forma, destaque na esfera internacional sobre essa temátical].

Os dados da Pesquisa Nacional por Amostra de Domicilio (PNAD), de 2013, comprovaram uma tendência positiva na SAN em todo território brasileiro entre os anos de 2004 e 2013. Segundo seus resultados, o acesso frequente e perdurável aos alimentos estava assegurado a mais de $77 \%$ dos domicílios do Brasil, em comparação a $65 \%$ em 2004[9], concluindo que cerca de 40 milhões de brasileiros passaram à condição de SAN em uma década. Em 2004, dos 26 estados e Distrito Federal, 16 tinham, aproximadamente, $8 \%$ da sua população em Insegurança Alimentar Grave, ao passo que em 2013, nenhum estado brasileiro encontrava-se nessa situação ${ }^{[10]}$.

Quanto à Sergipe, a PNAD (2013) mostrou o aumento da prevalência de SAN: em 2009 era 59,7\% e, em 2013, passou para 67,3\%, apresentando, ainda, taxa inferior à média nacional[9].

De acordo com os resultados apresentados pelo relatório intitulado The State of Food Insecurity in the World, publicado pela Food and Agriculture Organization of the United Nations ${ }^{11]}$, o Brasil saiu do mapa da fome, situação que perdurou durante anos em expressiva parcela da população. Porém, é de suma importância afirmar que, mesmo com resultados positivos no confronto à extrema pobreza e à fome, no âmbito da SAN, ainda há a carência de fortalecimento do SISAN, tanto para evitar retrocessos, como por ainda haver resultados a serem conquistados, como o pleno DHAA[12]. 
Assim, o presente estudo teve como objetivo avaliar a capacidade instalada para implantação e/ou fortalecimento do SISAN em municípios selecionados de Sergipe, visando propor adequações e recomendações necessárias. Justifica-se a sua realização pela necessidade de acompanhar e avaliar os resultados das ações de SAN, por ser uma demanda do Conselho Estadual de Segurança Alimentar e Nutricional e pela inexistência de estudos relativos a esse tema em Sergipe. Ressalta-se a relevância de fortalecer o SISAN nos municípios que já o aderiram, e implantar naqueles que ainda não aderiram, considerando que, por meio desse sistema, será exequível o monitoramento das ações e dos programas de SAN no nível municipal.

\section{METODOLOGIA}

Foi realizado um estudo transversal descritivo, de natureza quantitativa, com coleta de dados direta e pesquisa documental, em cinco municípios do estado de Sergipe. Após contato prévio realizado que envolveu o convite para participação da pesquisa, explicação sobre seus objetivos e agendamento para a coleta de dados, foram realizadas entrevistas com os principais atores sociais de $\mathrm{SAN}$ dos municípios que envolveram presidentes de Conselhos Municipais de SAN e gestores ligados a SAN dos municípios participantes. O agendamento das entrevistas com representantes dos municípios e os deslocamentos foram executados com o apoio do Conselho Estadual de Segurança Alimentar e Nutricional (CONSEAN) de Sergipe, durante o período de junho a julho/2015.

Durante as entrevistas buscou-se complementar as informações não encontradas na coleta indireta, que foi realizada por meio de pesquisas em sites oficiais dos órgãos públicos (federal, estadual e municipal) envolvidos na temática da SAN: Ministério do Desenvolvimento Social e Combate à Fome, Ministério da Saúde, Instituto Brasileiro de Geografia e Estatística (IBGE), e do Departamento de Processamento de Dados do Sistema Único de Saúde (DATASUS).

O estado de Sergipe, cuja capital é Aracaju, está localizado no nordeste brasileiro, conta com 75 municípios e possui (em 2015) uma população estimada de 2.242.937 habitantes ${ }^{[13]}$. Os cinco municípios foram selecionados intencionalmente, por demanda do CONSEAN/SE, parceiro na pesquisa. Foram eleitos: Umbaúba e Lagarto, que já tinham aderido ao SISAN; e Aracaju, Aquidabã e Canindé de São Francisco, sem adesão ao referido sistema. No momento da pesquisa para o estado de Sergipe a informação é que 3 municípios aderiram ao SISAN.

Umbaúba, que em 6 de fevereiro de 1954 foi elevada à categoria de cidade, fica a $81,83 \mathrm{~km}$ da capital de Sergipe, possui uma população estimada em 2016 de 24.832 pessoas e tem como unidade territorial 118,856 $\mathrm{km}^{2}$, Lagarto foi elevada à condição de cidade em 1880, possui como área da unidade territorial $968,921 \mathrm{~km}^{2}$ os biomas presentes nesse município são caatinga e mata atlântica. Canindé de São Francisco, banhado pelo Rio São Francisco possui uma população estimada em 2016 de 28.832 pessoas, sua área total é calculada em 902, 247 $\mathrm{km}^{2}$ e seu bioma é a caatinga. Já Aquidabã, município criado em 1882, possui como bioma a mata atlântica com uma área estimada em 359,005 $\mathrm{km}^{2}$ e uma população de 21.452 habitantes [13].

Foram utilizados dois instrumentos para cumprir os objetivos propostos: um check list para investigação da capacidade instalada para implantação e/ou fortalecimento do Sistema de Segurança Alimentar e Nutricional (SISAN), adaptado do modelo proposto pelo órgão gestor nacional doSISAN[G]; e uma matriz de indicadores para Avaliação da Segurança Alimentar e Nutricional dos municípios, adaptada de uma dissertação de mestrado, da Universidade Federal da Bahia ${ }^{[14]}$.

\section{Check List}

O check list foi composto por uma lista de 19 programas de SAN do Governo Federal para que o respondente informasse se o município participava ou não do programa, a unidade de administração e o nome do programa no local, se diferisse do nome proposto pelo Governo Federal. Caso o município tivesse algum programa (federal ou local) que não constasse na lista, este poderia ser registrado.

A última parte do check list foi composta por 13 perguntas relativas aos pressupostos para o SISAN: existência do CONSEA municipal, do SISAN, da CAISAN e Conferências de SAN realizadas nos municípios, bem como a participação da iniciativa privada, e realização de ações específicas para Povos e Comunidades Tradicionais. 
Capacidade instalada e SISAN em Sergipe. Silva, Fagundes e Silva

\section{Matriz de indicadores}

A matriz foi composta pela análise de indicadores em quatro dimensões da SAN: 'disponibilidade de alimentos', 'acesso aos alimentos', 'consumo de alimentos', 'utilização biológica de alimentos'. Para aplicação nos municípios Sergipanos foi necessário mudar algumas nomenclaturas e acrescentar indicadores, como o número de moradores que nunca frequentaram a escola e o percentual da população residente na área rural e urbana.

Foi utilizada uma escala de valores e categorias para a avaliação da SAN dos municípios. Cada indicador da matriz recebeu uma pontuação de 0 a 10 , sendo quanto mais próximo do valor 0 , maior o risco de Insegurança Alimentar e Nutricional e quanto maior a aproximação do 10 , maior a garantia da SAN para cada atributo.

Considerando a amplitude e complexidade do conceito de SAN, a análise foi realizada agrupando os indicadores de acordo com a abrangência do tema. A dimensão 'disponibilidade de alimentos' foi composta por dez indicadores que faziam referência ao transporte, à produção e à comercialização de alimentos em cada município, fatores que podem restringir a oferta de alimentos aos habitantes dos municípios. A dimensão 'acesso aos alimentos' foi composta por cinco indicadores que faziam alusão aos aspectos socioeconômicos e culturais que podem interferir no acesso aos alimentos. A dimensão 'consumo de alimentos' possuía três indicadores ligados às condições de saúde e nutrição, e a dimensão 'utilização biológica de alimentos' foi composta por seis indicadores associados às condições de acesso aos serviços sociais, de saneamento básico e de saúde, que podem interferir na utilização dos nutrientes ${ }^{[14]}$.

Os indicadores foram calculados para cada município mensurando as quatro dimensões, com pontuação máxima de 240 pontos. Após diversas buscas nas bases de dados, aqueles indicadores indisponíveis foram retirados do cálculo da média e foram classificados na pesquisa com a sigla 'SD' (sem dado), visando à sinalização de intervenções para melhorias aos responsáveis. A partir da pontuação máxima de cada município que foi obtida de acordo com os dos dados disponíveis foi realizado o cálculo proporcional para obtenção da prevalência (Quadro 1), classificando o município em uma das categorias de SAN, conforme os valores previamente definidos por Panelli-Martins (2007):

Quadro 1. Classificação das categorias de SAN. Pesquisa Fortalecimento do SISAN, Sergipe, 2015

\begin{tabular}{|c|l|l|}
\hline Percentual dos pontos & \multicolumn{1}{|c|}{ Classificação } & \multicolumn{1}{|c|}{ Definição } \\
\hline$\geq 75$ & Segurança alimentar e nutricional - SAN & $\begin{array}{l}\text { Representa uma situação ideal, na qual um conjunto de } \\
\text { fatores das dimensões atuou para garantir a realização do } \\
\text { conceito de SAN. }\end{array}$ \\
\hline 50 a 74,9 & Risco de insegurança alimentar e nutricional - RI & $\begin{array}{l}\text { Representa uma situação onde alguns fatores das } \\
\text { dimensões consideradas comprometem a realização plena } \\
\text { da SAN, porém sem riscos de privação alimentar } \\
\text { continuada e fome. }\end{array}$ \\
\hline $25 \%$ a 49,9 & Insegurança alimentar e nutricional leve - IL & $\begin{array}{l}\text { Representa uma situação onde alguns fatores das } \\
\text { dimensões comprometem a realização plena da SAN, } \\
\text { com relativa vulnerabilidade à privação alimentar e à } \\
\text { fome. }\end{array}$ \\
\hline $0,1 \%$ a 24,9 & Insegurança alimentar e nutricional moderada - IM & $\begin{array}{l}\text { Representa uma situação em que um maior número de } \\
\text { fatores das dimensões compromete a realização plena da } \\
\text { SAN, caracterizando uma situação de maior } \\
\text { vulnerabilidade à privação e à fome. }\end{array}$ \\
\hline 0 (zero) & Insegurança alimentar e nutricional grave - IG & $\begin{array}{l}\text { Representa uma situação em que um maior número de } \\
\text { fatores das dimensões compromete a realização plena da } \\
\text { SAN, caracterizando uma situação de privação e fome. }\end{array}$ \\
\hline
\end{tabular}

Fonte: Panelli-Martins, 2007, adaptado. 
A análise de dados de ambos os instrumentos foi descritiva, com frequências simples e relativas, realizadas por meio do programa Excel $^{\circledR}$ e do Software Statistical Package for the Social Sciences (SPSS), versão 17 para Windows.

A pesquisa foi realizada após a aprovação pelo Comitê de Ética em Pesquisa, da Universidade Federal de Sergipe (UFS), sob o parecer no 43485615.3.0000.554. Todos os participantes assinaram o Termo de Consentimento Livre e Esclarecido e o Termo de Consentimento Pós-Esclarecido.

\section{RESULTADOS E DISCUSSÃO}

No momento da entrevista com o representante do município A o Conselho Municipal de Segurança Alimentar e Nutricional, instância designada para apontar um respondente, estava há quatro meses em processo de mudança de gestão. Com o afastamento do presidente anterior e recém-posse do novo representante, este alegou não ser possível participar, por não ter a apropriação adequada sobre os programas e as ações de SAN no município. Informou que ainda não havia sido realizada a eleição dos conselheiros, e, portanto, não estavam sendo realizadas as reuniões periódicas mensais. Visando obter informações sobre o objeto principal da pesquisa foi questionada a situação do SISAN no município, e o respondente informou não ter conhecimento.

Após buscar sem sucesso a indicação de outro respondente e considerando que duas dimensões da matriz de indicadores teriam que ser respondidas por um informante-chave, os pesquisadores decidiram por não contemplar o município em questão, dada a situação vivenciada e a falta de informações. Considerase, porém, que essa situação já é, por si, um expressivo resultado de pesquisa, que mostrou desarticulação e um processo moroso de troca de gestão, capaz de comprometer a situação de SAN da população do município A. A ausência de informações aponta para uma fragilidade da capacidade instalada para o SISAN no município.

Desse modo, os resultados apresentados referem-se aos outros 4 municípios participantes da pesquisa (B, C, D e E). Os gestores foram questionados quanto ao funcionamento de 19 programas sociais nos municípios (Quadro 2), sendo que oito não existiam em nenhum dos municípios avaliados. A ausência de alguns pode ser justificada pela não contemplação do município nos parâmetros para adesão (número de habitantes, por exemplo) ou pela falta de articulação da gestão, o que pode interferir na consolidação da SAN a sua população.

Os oito programas ausentes, chamaram atenção devido ao incentivo de implantação por parte do Governo Federal e aos potenciais impactos relatados: o Consórcio de Segurança Alimentar e Desenvolvimento Local (CONSAD), que visa à articulação regional com o intuito de agregar ações que promovam o desenvolvimento local e a SAN; os Restaurantes Populares e as Cozinhas Comunitárias, que têm como objetivo ofertar refeições nutricionalmente adequadas a um custo mais acessível à população; o Programa Banco de Alimentos, que contribui para o abastecimento em entidades sociais; os Programas de Agricultura Urbana e Periurbana e de Alimentação do Trabalhador, que têm o objetivo de estimular o consumo de alimentos saudáveis e a Unidade de Beneficiamento e Processamento Familiar e Agroalimentar, que visa agregar valor aos diversos produtos das comunidades rurais, urbanas e periurbanas carentes ${ }^{[15]}$, todos de extrema importância para auxiliar na redução da Insegurança Alimentar local.

Os municípios $\mathrm{D}$ e $\mathrm{E}$ apresentaram o Programa de Aquisição de Alimentos da Agricultura Familiar (PAA), fator positivo no tocante ao combate da Insegurança Alimentar e Nutricional. De acordo com Grisa et al.[16] o PAA abrange tanto as necessidades de acesso aos alimentos das populações, como as demandas dos agricultores familiares de conquistar mercados para seus produtos. Segundo Brasil[ ${ }^{[17]}$ esse programa tem como objetivo a valorização e o estímulo à atividade da agricultura familiar, garantindo às populações em situação de insegurança o acesso aos alimentos em quantidade e qualidade suficientes, além de fortalecer a agricultura familiar no campo e pequeno agricultor. A existência de Mercados Populares em todos os municípios, bem como a presença de feiras livres nessas cidades são estratégias importantes para a promoção da SAN. Conforme os documentos oficiais da presidência da Repúblicaa ${ }^{[15]}$ afirmam, elas estimulam a diversificação da produção, aumento das demandas de produtos agroecológicos para a ampliação dos sistemas locais e são equipamentos importantes para valorização das culturas e tradições familiares. 
A Educação Alimentar e Nutricional (EAN) se insere na esfera das políticas públicas no âmbito da SAN $[17]$. Os respondentes dos municípios $\mathrm{B}$ e $\mathrm{D}$ relataram realizar ações de Educação Alimentar e Nutricional à população, as quais de acordo com documentos oficiais da república ${ }^{[18]}$ são consideradas fundamentais para transmitir conhecimentos e práticas contínuas, permanentes, autônomas e voluntárias, de hábitos alimentares saudáveis, para a promoção do DHAA e garantia de SAN.

O Programa Cisternas fomenta as condições de acesso à água para o consumo e para a produção de alimentos, destinado às famílias rurais de baixo nível socioeconômico e que não possuem meios disponíveis para captação e armazenamento de água, ou que tenham acesso irregular ou precário à água para produção e consumo ${ }^{[19]}$. O município $C$, segundo o respondente da pesquisa não possuía o Programa Cisternas. Esse resultado é um indicador negativo, devido à sua localização geográfica e bioma, os quais fazem dele um município carente de água, em especial nos tempos de seca. Embora o gestor tenha afirmado a ausência do programa no município, de acordo com os documentos oficiais da presidência da República ${ }^{[20]} \mathrm{em}$ Sergipe, até aquele ano a Companhia de Desenvolvimento dos Vales do São Francisco e do Parnaíba (CODEVASF), havia instalado 1.346 cisternas em locais como o município em questão, Gararu, Itabi, Japaratuba, entre outros.

Quadro 2. Presença de programas e ações federais nos municípios e a capacidade instalada. Pesquisa Fortalecimento do SISAN, Sergipe, 2015

\begin{tabular}{|c|c|c|c|c|}
\hline Programas e ações federais & $\begin{array}{c}\text { Município } \\
\text { B }\end{array}$ & $\begin{array}{c}\text { Município } \\
\text { C }\end{array}$ & $\begin{array}{c}\text { Município } \\
\text { D }\end{array}$ & $\begin{array}{c}\text { Município } \\
\mathrm{E}\end{array}$ \\
\hline Programa Bolsa Família & $\mathrm{X}$ & $\mathrm{X}$ & $\mathrm{X}$ & $\mathrm{X}$ \\
\hline Programa de Aquisição de Alimentos da Agricultura Familiar & & & $\mathrm{X}$ & $\mathrm{X}$ \\
\hline Mercados Populares & $\mathrm{X}$ & $\mathrm{X}$ & $\mathrm{X}$ & \\
\hline Feiras Livres & $\mathrm{X}$ & $\mathrm{X}$ & $\mathrm{X}$ & $\mathrm{X}$ \\
\hline Sistema de Segurança Alimentar e Nutricional (SISAN) & & & $\mathrm{X}$ & $\mathrm{X}$ \\
\hline Programa Nacional de Alimentação Escolar (PNAE) & $\mathrm{X}$ & $\mathrm{X}$ & $\mathrm{X}$ & $\mathrm{X}$ \\
\hline Educação Alimentar e Nutricional & $\mathrm{X}$ & & $\mathrm{X}$ & \\
\hline Programa Cisterna & $\mathrm{X}$ & & $\mathrm{X}$ & \\
\hline PRONAF - Garantia Safra & $\mathrm{X}$ & $\mathrm{X}$ & $\mathrm{X}$ & $\mathrm{X}$ \\
\hline PRONAF - Seguro AF & $\mathrm{X}$ & $\mathrm{X}$ & $\mathrm{X}$ & $\mathrm{X}$ \\
\hline Cesta Básica & $\mathrm{X}$ & $\mathrm{X}$ & & \\
\hline Existência de associações ou cooperativa para produção de alimentos & & $\mathrm{X}$ & $\mathrm{X}$ & $\mathrm{X}$ \\
\hline Percentagem da quantidade de programas & 52,9 & 47,0 & 57,8 & 47,0 \\
\hline Capacidade instalada e ações realizadas & $\begin{array}{c}\text { Município } \\
\text { B }\end{array}$ & $\begin{array}{c}\text { Município } \\
\mathrm{C}\end{array}$ & $\begin{array}{c}\text { Município } \\
\text { D }\end{array}$ & $\begin{array}{c}\text { Município } \\
\mathrm{E}\end{array}$ \\
\hline Iniciativa de empresas privadas & & & $\mathrm{X}$ & \\
\hline Presença do CONSEA Municipal & $\mathrm{X}$ & $\mathrm{X}$ & $\mathrm{X}$ & $\mathrm{X}$ \\
\hline CAISAN & $\mathrm{X}$ & $\mathrm{X}$ & $\mathrm{X}$ & $\mathrm{X}$ \\
\hline Realização de Conferências de SAN & $\mathrm{X}$ & $\mathrm{X}$ & $\mathrm{X}$ & \\
\hline Participação/Realização de Reuniões Territoriais ou Ampliadas & $\mathrm{X}$ & $\mathrm{X}$ & & \\
\hline Presença de Povos e Comunidades Tradicionais & $\mathrm{X}$ & $\mathrm{X}$ & $\mathrm{X}$ & $\mathrm{X}$ \\
\hline Ações de SAN específicas para Povos e Comunidades Tradicionais & $\mathrm{X}$ & & & \\
\hline
\end{tabular}

Todos os municípios apresentaram o Programa Bolsa Família (PBF), o qual conforme Cotta e Machado ${ }^{[21]}$ foi instituído pelo governo brasileiro para transferência direta e condicionada de renda. De acordo com Monteiro et al.[2] o programa foi criado em 2003 de modo a combater, de forma imediata, a fome e a miséria, buscando promover a SAN e diminuir a desigualdade social do país. Os programas de 
transferência de renda têm alcançado destaque como políticas de proteção social e combate à pobreza em diversos países, inclusive no Brasil[21]. Dados de setembro de 2015 revelam que 13,9 milhões de famílias são beneficiadas pelo PBF no país, número que está sendo mantido desde 2012[22].

O Programa Nacional de Alimentação Escolar (PNAE) é vigente em todos os municípios da pesquisa. Conforme Saraiva et al.[23] a alimentação escolar é afirmada como um direito dos escolares e considerada como uma estratégia de SAN. Com atuação voltada para a redução máxima do total de pessoas em situação de Insegurança Alimentar no país ${ }^{[24]}$ o programa foi implantado em 1955, contribuindo até hoje para a efetivação da SAN, por meio do crescimento, desenvolvimento, aprendizagem, rendimento escolar e formação de hábitos alimentares saudáveis ${ }^{[2]}$.

O Programa Nacional de Agricultura Familiar (Pronaf) está presente em todos os munícipios participantes da pesquisa, o que representa uma perspectiva positiva para a SAN. Os documentos oficiais da república[24] afirmam que se trata de uma política pública de produção e disponibilidade de alimentos, o documento oficial da presidência da República ${ }^{[2]}$ coaduna que o programa é destinado a fomentar a geração de renda e melhorar o uso da mão de obra familiar por meio do financiamento de atividades e serviços rurais.

Os respondentes da pesquisa foram questionados quanto às diretrizes relacionadas às ações de SAN nos municípios, tais como a presença ou ausência de projetos e participação da iniciativa privada nos municípios, a existência do Conselho de Segurança Alimentar e Nutricional no município, como se dá o funcionamento do mesmo, a existência do SISAN no município, a existência da CAISAN, a realização ou não de alguma conferência, o número de conferências realizadas até o momento da pesquisa, presença de Povos e Comunidades Tradicionais (PCT) e se existiam ações específicas para essa população (Quadro 2).

Todos os municípios participantes da pesquisa possuem o Conselho Municipal de Segurança Alimentar (CONSEAM), o qual de acordo com Brasil[27] é um órgão de articulação entre a sociedade civil e governo, que estimula a população a participar da formulação, execução e acompanhamento de políticas de SAN, sendo de suma importância para as conquistas sociais e superação da pobreza.

A CAISAN estava presente em todos municípios, a mesma tem a função de fortalecer a intersetorialidade, pois é a instância que possibilita a inserção e a integração dos diferentes setores os quais são responsáveis pelas políticas públicas e programas de SAN. Apesar da presença do CONSEAM em todos os municípios participantes da pesquisa, três municípios não aderiram ao SISAN, o que consoante com o documento oficinal da Repúblical[28] faz com que percam força política pois, o mesmo intercede por políticas de SAN de forma intersetorial e integrada com a sociedade civil a nível local.

Outro requisito para o SISAN é a realização de Conferências de SAN, não referida apenas pelo respondente do município $\mathrm{E}$, o que aponta para um possível distanciamento entre os gestores e a sociedade civil, já que essas instâncias de representação popular buscam a ampliação e o fortalecimento dos compromissos políticos para a promoção da Soberania Alimentar e garantia ao DHAA. A LOSAN registra que a execução do DHAA e da SAN solicita o respeito à Soberania Alimentar a qual concede aos países prioridade de suas decisões sobre o consumo e a decisão de alimentos[3]. As conferencias de SAN apresentam a proposta de Soberania Alimentar por levar em consideração que os países devam ser soberanos para que dessa forma possam promover a SAN de seus povos (Soberania Alimentar), respeitando suas diversas peculiaridades culturais, refletidas no ato de comer ${ }^{[1]}$.

Todos os municípios da pesquisa possuem Povos e Comunidades Tradicionais (PCT) em seus territórios geográficos, porém apenas o respondente do município B relatou possuir ações de SAN específicas para essas populações. Ressalta-se que em conformidade com o documento oficial do Brasil[15] é uma recomendação nacional retirar os PCT da invisibilidade, por meio de reconhecimento, autoafirmação e garantia dos seus direitos quanto a território, economia, sociedade e cultura, buscando a valorização da sua identidade [29]. O relatório da FAO aponta como situação prioritária, o enfrentamento da situação vulnerável crônica dos $\mathrm{PCT}^{[24]}$, pois o diagnóstico da situação de SAN dos PCT defronta com obstáculos associados à histórica exclusão social e a invisibilidade a que enfrentaram, impedindo melhor 
percepção de suas condições alimentares e necessidades ainda nos dias de hoje ${ }^{[10]}$.

Quanto a matriz de indicadores, foram encontradas situações de agravos que podem comprometer a realização integral da SAN[30], como acesso aos alimentos e utilização biológica dos alimentos (Tabela 1).

$\mathrm{O}$ município $\mathrm{E}$ foi o único que não apresentou, na dimensão de disponibilidade, ação no indicador 'práticas sustentáveis de produção de alimentos saudáveis', o qual segundo Schutter ${ }^{[31]}$ é fundamental por proporcionar a disponibilidade de alimento à população, aumentar a renda dos pequenos agricultores e por não comprometer a capacidade da terra em garantir as necessidades futuras, não atingindo a biodiversidade, a água e as temperaturas do planeta, aspectos fundamentais para a SAN a qual aborda também a temática da sustentabilidade.

$\mathrm{O}$ indicador 'cooperativas de agricultores familiares' era inexistente no município de B, o que de acordo com Chiarello[32] acaba por desfavorecer o desenvolvimento do mercado local, a produção em pequenas propriedades o alcance a economias de escala e a facilidade na ação de agregar valor aos alimentos produzidos $^{[31]}$, sendo mais um agravo para a disponibilidade de alimentos.

$O$ indicador 'acesso à terra' é condição essencial, por possibilitar o plantio para autoconsumo. Estudos realizados com populações do campo relatam um maior déficit nutricional associado à diminuição da posse de terra[3]. Três dos quatros municípios estudados alegaram acesso à terra na forma propriedade privada, e o município B alegou arrendamento no município, fatores que minimizam as possibilidades para os pequenos agricultores, comprometendo a SAN das famílias locais.

No que se relaciona à dimensão 'acesso aos alimentos', o indicador de população residente em área urbana e rural mostrou maior prevalência da população dos municípios na área urbana, sendo B com $62,0 \%, \mathrm{E}$ $\operatorname{com} 62,0 \%, C \operatorname{com} 56,9 \%$ e D com 51,5\% ${ }^{[34]}$. No Brasil, os domicilios rurais aglomeram condições sociais inadequadas, como rendimento médio familiar mais baixo, superior informalidade nas relações de trabalho, baixa escolaridade, entre outros, o que acabam por levar a uma maior prevalência de Insegurança Alimentar nessa região[35].

Alguns fatores que contribuíram para o Risco de Insegurança Alimentar e Nutricional encontrado no município $\mathrm{C}$ foram o elevado número de moradores por domicilios particulares (maior diluição de renda) e o percentual de famílias chefiadas por mulheres, que influenciam na renda familiar e reduz o potencial de compra dos alimentos ${ }^{[3]}$. De acordo com o Censo Demográfico de 2010[36] levando em consideração a variável renda ao analisar essa variável por sexo, foi possível identificar que os homens possuem rendimento maior que as mulheres, esse fato se repete quando visto ao nível de Brasil, como a nível de Nordeste, fator que pode estar ligado a maior probabilidade de Insegurança Alimentar e domicílios chefiados por mulheres.

Nos municípios B e D também foi identificado alto percentual de domicílios chefiados por mulheres [34]. Dados nacionais revelam que 79,0\% dos domicilios que têm como responsáveis os homens estão em SAN já, quando as mulheres assumem a chefia do domicilio esse número cai para $75,0^{\%} 0^{[10]}$, mostrando uma diversidade da condição de SAN por gênero.

No município C, o resultado identificado na dimensão 'consumo de alimentos' teve influência do indicador de aleitamento materno exclusivo, o mesmo encontrava-se abaixo da média nacional (41,0\%), contudo, segundo os parâmetros da matriz de indicadores de SAN, proposta pela Universidade Federal da Bahia, foi classificado como adequado $(30,7 \%)$. O indicador aleitamento materno teve como resultado a inadequação com o percentual de 18,3\%[3] no município D, o mesmo é uma estratégia importante de SAN pois, é uma prática capaz de proteger lactentes que moram em residências com Insegurança Alimentar em virtude de o leite materno não apresentar custo, além de possuir efeito positivo na saúde, no estado nutricional e no desenvolvimento das crianças ${ }^{[38]}$. 
Capacidade instalada e SISAN em Sergipe. Silva, Fagundes e Silva

Tabela 1. Pontuação dos municípios participantes sobre a SAN. Pesquisa Fortalecimento do SISAN, Sergipe, 2015

\begin{tabular}{|c|c|c|c|c|c|c|c|}
\hline \multirow{2}{*}{\multicolumn{2}{|c|}{ Municípios }} & \multicolumn{4}{|c|}{ Dimensão } & \multicolumn{2}{|c|}{ Total } \\
\hline & & \multirow{2}{*}{$\begin{array}{c}\text { Disponibilidade } \\
100,0\end{array}$} & \multirow{2}{*}{$\begin{array}{c}\text { Acesso } \\
30,0\end{array}$} & \multirow{2}{*}{$\begin{array}{c}\text { Consumo } \\
20,0\end{array}$} & $\begin{array}{l}\text { Utilização } \\
\text { biológica }\end{array}$ & Pontos & \multirow[t]{2}{*}{$\%$} \\
\hline \multirow{2}{*}{ Município B } & $\begin{array}{l}\text { Pontuação } \\
\text { máxima }\end{array}$ & & & & 40,0 & 190,0 & \\
\hline & $\begin{array}{c}\text { Pontuação } \\
\text { Obtida }\end{array}$ & 51,0 & 10,0 & 15,0 & 25,0 & 101,0 & 53,1 \\
\hline \multirow{2}{*}{ Município C } & $\begin{array}{c}\text { Pontuação } \\
\text { máxima }\end{array}$ & 100,0 & 40,0 & 30,0 & 60,0 & 230,0 & \\
\hline & $\begin{array}{c}\text { Pontuação } \\
\text { Obtida }\end{array}$ & 71,0 & 10,0 & 20,0 & 35,0 & 136,0 & 59,1 \\
\hline \multirow{2}{*}{ Município D } & $\begin{array}{c}\text { Pontuação } \\
\text { máxima }\end{array}$ & 100,0 & 40,0 & 20,0 & 40,0 & 200,0 & \\
\hline & $\begin{array}{c}\text { Pontuação } \\
\text { Obtida }\end{array}$ & 86,0 & 20,0 & 5,0 & 20,0 & 131,0 & 65,5 \\
\hline \multirow{2}{*}{ Município E } & $\begin{array}{l}\text { Pontuação } \\
\text { máxima }\end{array}$ & 100,0 & 30,0 & 20,0 & 40,0 & 190,0 & \\
\hline & $\begin{array}{c}\text { Pontuação } \\
\text { Obtida }\end{array}$ & 64,0 & 20,0 & 20,0 & 20,0 & 124,0 & 65,2 \\
\hline \multirow[t]{2}{*}{ Total } & $\begin{array}{l}\text { Pontos } \\
\text { obtidos }\end{array}$ & 272,0 & 60,0 & 60,0 & 100,0 & - & - \\
\hline & $\%$ & 68,0 & 42,8 & 66,6 & 55,5 & - & - \\
\hline
\end{tabular}

O resultado encontrado na dimensão 'utilização biológica dos alimentos' teve contribuição da deficiente cobertura de saneamento básico, incluindo o acesso à água tratada nas residências. $\mathrm{O}$ município $\mathrm{C}$ com $78,8 \%$, o D com $73,4 \%$, e o E com $32,0 \%$ da população com acesso à água trata, foram classificados quanto a esse indicador como inadequados, já o município B que apresentou $81,4 \%$ foi classificado como aceitável da população com acesso à água por rede geral, em conformidade com os parâmetros da matriz de indicadores de SAN, proposta pela Universidade Federal da Bahia, adaptada à realidade do estado de Sergipe ${ }^{[34]}$.

O esgotamento sanitário apresentou-se inadequado nos municípios $\mathrm{B}$ e $\mathrm{C}$, e a coleta de lixo doméstico foi caracterizada como aceitável em concordância com Panelli-Martins et al.[30]. Os dados encontrados para coleta de lixo doméstico foram: $\mathrm{E}$ $81,1 \%$, C 79,7\%, B 73,8\%, e D 72,7\% [34]. As medidas de saneamento básico garantem melhores condições de saúde para os indivíduos, e estão associadas à adequada utilização dos nutrientes, compreendida como pleno aproveitamento biológico dos alimentos ${ }^{[38]}$.

Devido à falta de atualização e alimentação nos bancos de dados públicos, alguns indicadores ficaram sem os resultados no momento da pesquisa: a renda do responsável pelos domicilios particulares, indisponível para todos os municípios; o número de moradores por domicílios particulares, no município B; o percentual de familias chefiadas por mulheres, no município $\mathrm{E}$.

Recomenda-se mais investimento na construção de armazéns e/ou depósitos municipais para estoque de alimentos, visando garantir o suprimento do mercado interno; aumento e incentivo a ações voltadas para alimentação saudável nas escolas, nas unidades de saúde, nas associações de moradores; incentivo à prática sustentável de alimentos saudáveis, mostrando a importância da produção para autoconsumo, ressaltando a produção sem o uso de agrotóxicos. Sugere-se atividades de valorização dos orgânicos e feiras da agricultura orgânica, a criação de cooperativas para pequenos agricultores favorecendo a oferta local de alimentos e contribuindo na renda familiar dos pequenos agricultores. É fundamental também o investimento em saneamento básico nos municípios, incluindo o acesso à água tratada nas residências, esgotamento sanitário e coleta de lixo para todos, garantindo assim melhores condições de saúde aos indivíduos. 


\section{CONCLUSÕES}

A identificação do Risco de Insegurança Alimentar em todos os municípios participantes mostra que a capacidade instalada para o SISAN ainda é frágil, mesmo nos municípios que já o implantaram. O estudo revelou lacunas em ações necessárias e o não alcance dos seus objetivos. É preciso maior atenção por parte dos gestores e da sociedade civil ao SISAN, para que o mesmo possa ser sustentado, contribuindo para o acesso pleno à alimentação adequada como direito essencial do indivíduo.

Nos municípios onde o sistema ainda não foi implantado é preciso esforço por parte dos gestores, na busca a atender aos requisitos mínimos necessários para a sua implantação. É recomendado o estímulo à atuação concreta dos Conselhos Municipais em todos locais participantes da pesquisa, tendo em vista os relatos de que ainda há certa resistência por parte dos membros para atuação nesta instância do controle social. É premente estimular a sociedade civil quanto à importância da sua inserção e atuação nos Conselhos Municipais de SAN para que possam ser ouvidas e trabalhadas as demandas locais.

Foi estratégica a utilização dos dois instrumentos para a coleta de dados, pois trouxeram dados complementares (indicadores populacionais e de políticas públicas e serviço) sobre a situação de SAN e do andamento do SISAN nos municípios.

O estudo realizado apresentou limitações como a dificuldades quanto à coleta de dados, uma vez que nem sempre os indicadores estavam disponíveis ou eram dados antigos, deixando claro que ainda é preciso avançar na alimentação e atualização das bases de dados disponíveis para toda população. Outra limitação é a análise não apresentar características sobre a qualidade das políticas públicas implementadas, mas sim o fato de elas existirem ou não. Trabalhos futuros poderão preencher esse tipo de lacuna.

Sugere-se a continuidade desse tipo de avaliação e ampliação do estudo para outros municípios do estado de Sergipe.

\section{AGRADECIMENTOS}

Aos participantes da pesquisa. Ao CONSEAN Sergipe, pelo apoio e parceria permanentes. Ao PIBICUFS, pela Bolsa de Iniciação Científica.

\section{REFERÊNCIAS}

[1] Burity V, Franceschini T, Valente FLS, Recine E, Leão M, Carvalho MF. Direito humano à alimentação adequada no contexto da segurança alimentar e nutricional. Brasilia (DF): Abrandh; 2010.

[2] Monteiro F, Schmidt ST, Costa IB, Almeida CCB, Matuda NS. Bolsa Família: insegurança alimentar e nutricional de crianças menores de cinco anos. Ciênc. Saúde Coletiva. 2014;19(5):13471357.

[3] Brasil. Lei no 11.346, de 15 de setembro de 2006. Cria o Sistema Nacional de Segurança Alimentar e Nutricional - SISAN com vistas em assegurar o direito humano à alimentação adequada e dá outras providências. Diário Oficial da União. 18 set 2006.

[4] Brasil. Conselho Nacional de Segurança Alimentar e Nutricional (CONSEA). A Segurança Alimentar e Nutricional e o Direito Humano à Alimentação Adequada no Brasil [Internet]. 2010 [acesso em 01 jun 2015]. Disponível em: http://www4.planalto.gov.br/consea/publicacoes/a-segurancaalimentar-e-nutricional-e-o-direito-humano-a-alimentacaoadequada-no-brasil-1/relatorio-consea.pdf

[5] Ministério do Desenvolvimento Social e Combate à Fome. Sistema Nacional de Segurança Alimentar e Nutricional - SISAN: Diagnóstico de Implantação no Âmbito Estadual [Internet]. 2010 [acesso em 01 jun 2015]. Disponível em: http://www.sesc.com.br/mesabrasil/doc/Documento-base-

Diagnostico-SISAN.pdf

[6] Brasil. Conselho Nacional de Segurança Alimentar e Nutricional. Sistema Nacional de Segurança Alimentar e Nutricional [Internet]. Brasilia (DF): 2013 [acesso em 17 jan 2016]. Disponível em: http://www4.planalto.gov.br/consea

[7] Ministério do Desenvolvimento Social e Combate à Fome Indicadores e Principais Resultados do Plano de Segurança Alimentar e Nutricional [Internet]. 2015 [acesso em 16 jan 2016]. Disponível em: http://issuu.com/informecaisan/docs

[8] Brasil. Conselho Nacional de Segurança Alimentar e Nutricional. Construção do Sistema e da Política Nacional de Segurança Alimentar e Nutricional: a experiência brasileira [Internet]. 2009 [acesso em 17 jan 2016]. Disponível em: https://www.fao.org.br

[9] Instituto Brasileiro de Geografia e Estatística - IBGE. Pesquisa Nacional por Amostra de Domicílio: Segurança Alimentar - 2013 [Internet]. Rio de Janeiro; 2014 [acesso em 15 nov 2015]. Disponível em: http://biblioteca.ibge.gov.br/visualizacao/livros/liv91984.pdf 
Capacidade instalada e SISAN em Sergipe. Silva, Fagundes e Silva

[10] Organização das Nações Unidas para Alimentação e a Agricultura (FAO). O Estado da Segurança Alimentar e Nutricional no Brasil: agendas convergentes [Internet]. Brasilia (DF); 2015 [acesso em 16 jan 2016]. Disponível em: http://issuu.com/informecaisan/docs/indicadoreseprincipaisresult adosdop

[11] Food and Agriculture Organization of the United Nations FAO. The State of Food Insecurity in the World 2014: Strengthening the enabling the enabling environment for food security and nutrition [Internet]. Rome; 2015. [acesso em 15 nov 2015]. Disponível em: http://www.fao.org/3/a-i4030e.pdf.

[12] Ministério do Desenvolvimento Social e Combate à Fome. Mapa SAN 2014: Mapeamento de Segurança Alimentar e Nutricional. Brasília (DF): Ministério do Desenvolvimento Social e Combate à Fome; 2015.

[13] Instituto Brasileiro de Geografia e Estatística. Estados: Sergipe [Internet]. Rio de Janeiro; 2015 [acesso em 02 fev 2017]. Disponível em: http://www.ibge.gov.br

[14] Panelli-Martins BE. Análise de método de avaliação da segurança alimentar e nutricional: uma contribuição à política municipal de SAN [dissertação]. Salvador: Universidade Federal da Bahia; 2007.

[15] Ministério do Desenvolvimento Social e Combate à Fome. Guia de políticas e programas do Ministério do Desenvolvimento Social e Combate à Fome - MDS. Brasília (DF): Ministério do Desenvolvimento Social e Combate à Fome; 2011.

[16] Grisa C, Schmitt JC, Mattei FL, Maluf RS, Leite SP. Contribuições do Programa de Aquisição de Alimentos à segurança alimentar e nutricional e à criação de mercados para a agricultura familiar. Agriculturas. 2011;8(3):34-41.

[17] Brasil. Lei no 12.512, de 14 de julho de 2011. Institui o Programa de Apoio à Conservação Ambiental e o Programa de Fomento às Atividades Produtivas Rurais; altera as Leis nos 10.696, de 2 de julho de 2003, 10.836, de 9 de janeiro de 2004, e 11.326, de 24 de julho de 2006. Diário Oficial da União. 14 jul 2011.

[18] Ministério do Desenvolvimento Social e Combate à Fome. Marco de referência de educação alimentar e nutricional para as políticas públicas [Internet]. Brasília (DF); 2012 [acesso em 30 mar 2016]. Disponível em: http://www.ideiasnamesa.unb.br

[19] Ministério do Desenvolvimento Social e Combate à Fome. Programa Cisternas - Água para beber e para agricultura [Internet]. Brasília (DF); 2016 [acesso em 01 mar 2016]. Disponível em: http://mdspravoce.mds.gov.br

[20] Ministério da Integração Nacional. Cisternas do Água para Todos já beneficiam mais de 1.300 famílias em Sergipe. Companhia de Desenvolvimento dos Vales do São Francisco e Parnaíba [Internet]. 2012 [acesso em 01 jun 2015]. Disponível em: http://www.codevasf.gov.br/noticias/2007/cisternas-do-aguapara-todos-ja-beneficiam-mais-de-1-300-familias-em-sergipe
[21] Cotta MMR, Machado JC. Programa Bolsa Família e segurança alimentar e nutricional no Brasil: revisão crítica da literatura. Rev Panam Salud Publica. 2013;33(1):54-60.

[22] Ministério do Desenvolvimento Social e Combate à Fome. Número de beneficiários do Bolsa Família tem se mantido estável [nternet]. Brasilia (DF); 2015 [acesso em 16 mar 2016]. Disponível em: http://mds.gov.br

[23] Saraiva EB, Silva APF, Sousa AA, Cerqueira GF, Chagas CMS, Toral N. Panorama da compra de alimentos da agricultura familiar para o Programa Nacional de Alimentação Escolar. Ciênc. Saúde Coletiva. 2013;1(4):927-936.

[24] Organização das Nações Unidas para Alimentação e a Agricultura (FAO). O Estado da Segurança Alimentar e Nutricional no Brasil: Um retrato multidimensional [Internet]. Brasilia (DF); 2014 [acesso em 16 dez 2015]. Disponível em: https://www.fao.org.br/download/SOFI_p.pdf

[25] Brasil. Lei no 11.947, de 16 de junho de 2009. Dispõe sobre o atendimento da alimentação escolar e do Programa Dinheiro Direto na Escola aos alunos da educação básica. Diário Oficial da União. 16 jun 2009.

[26] Ministério do Desenvolvimento Social e Combate à Fome. FAQ - Programa Nacional de Fortalecimento da Agricultura Familiar Pronaf. Banco Central do Brasil [Internet]. 2015 [acesso em 05 jan 2016]. Disponível em: http://www.bcb.gov.br

[27] Brasil. Como criar o Conselho Municipal de Segurança Alimentar e Nutricional [Internet]. Brasilia (DF): CONSEA; 2011 [acesso em 01 mar 2016]. Disponível em: http://www4.planalto.gov.br/consea/publicacoes/cartilha-comocriar-conseas-municipais

[28] Ministério do Desenvolvimento Social e Combate à Fome. Orientações para a adesão dos municípios ao SISAN. Brasilia (DF): Ministério do Desenvolvimento Social e Combate à Fome; 2014.

[29] Ministério do Meio Ambiente. Povos e Comunidades Tradicionais [Internet]. Brasília (DF); 2016 [acesso em 01 mar 2016]. Disponível em: http://www.mma.gov.br

[30] Panelli-Martins BE, Santos SMC, Assis AMO. Segurança Alimentar e nutricional: desenvolvimento de indicadores e experimentação em um município da Bahia, Brasil. Rev. Nutr. 2008;1(21):65-81.

[31] Schutter O. A agroecologia e o direito humano à alimentação adequada. Brasília (DF): Ministério do Desenvolvimento Social; 2012.

[32] Chiarello CL. Análise da gestão de cooperativas rurais tradicionais e populares: estudo de casos na Cocamar e Copavi [dissertação]. São Carlos: Universidade Federal de São Carlos; 2006. 
[33] Carneiro FF, Tambellini AT, Silva JÁ, Haddad AJP, Búrigo AC, Sá WR, et al. Saúde de famílias do Movimento dos Trabalhadores Sem Terra e de bóias-frias, Brasil, 2005. Rev. Saúde Pública. 2008;42(4):757-763.

[34] Instituto Brasileiro de Geografia e Estatística. Sergipe: Censo Demográfico 2010 [Internet]. Rio de Janeiro; 2010 [acesso em 04 jan 2016]. Disponível em: http://cidades.ibge.gov.br

[35] Instituto Brasileiro de Geografia e Estatística. Síntese de Indicadores Sociais: uma análise das condições de vida da população brasileira 2014 [Internet]. Rio de Janeiro; 2014 [acesso em 10 nov 2015]. Disponível em: http://biblioteca.ibge.gov.br

[36] Instituto Brasileiro de Geografia e Estatística. Indicadores Sociais Municipais: uma análise dos resultados do universo do Censo Demográfico 2010. Rio de Janeiro: IBGE; 2011.

[37] Ministério do Desenvolvimento Social e Combate à Fome. Pesquisa de Prevalência de Aleitamento Materno em Municípios Brasileiro: Situação do Aleitamento Materno em 227 municípios brasileiros [Internet]. Brasília (DF); 2010 [acesso em 10 nov 2015]. Disponível em: http://www.redeblh.fiocruz.br/media/pamuni.pdf

[38] Gomes GP, Gubert MB. Aleitamento materno em crianças menores de 2 anos e situação domiciliar quanto à segurança alimentar e nutricional. J Pediatr (Rio J). 2012;88(3):279-282. 
Capacidade instalada e SISAN em Sergipe. Silva, Fagundes e Silva

\section{APÊNDICE}

Check list
Data:

Entrevistador:

Pesquisa Capacidade instalada para fortalecimento da SAN

\section{ROTEIRO DE ENTREVISTA}

IDENTIFICAÇÃO DAS AÇÕES, PROGRAMAS E EQUIPAMENTOS SOCIAIS DE SAN NO MUNICÍPIO.

Nome completo do informante:

Município:

Instituição/ órgão:

\begin{tabular}{|c|c|c|c|c|c|c|c|c|c|}
\hline \multirow{2}{*}{ Programas do Governo Federal } & \multicolumn{2}{|c|}{$\begin{array}{c}\text { Existe/existiu no } \\
\text { município? }\end{array}$} & \multicolumn{2}{|c|}{ Ainda é vigente? } & \multirow{2}{*}{$\begin{array}{l}\text { Tempo de } \\
\text { existência } \\
\text { (em anos) }\end{array}$} & \multirow{2}{*}{$\begin{array}{c}\text { Unidade da } \\
\text { administração } \\
\text { municipal }\end{array}$} & \multirow{2}{*}{$\begin{array}{l}\text { Nome do } \\
\text { Programa no local }\end{array}$} & \multirow{2}{*}{ Avaliação ${ }^{5}$} & \multirow{2}{*}{ OBS } \\
\hline & Sim & Não & Sim & Não & & & & & \\
\hline \multicolumn{10}{|l|}{ 1. Programa Bolsa Família } \\
\hline \multicolumn{10}{|l|}{$\begin{array}{l}\text { 2. Programa de Aquisição de Alimentos da Agricultura } \\
\text { Familiar (PAA) - Doação Simultânea }\end{array}$} \\
\hline \multicolumn{10}{|l|}{$\begin{array}{l}\text { 3. Consórcio de Segurança } \\
\text { Desenvolvimento Local (CONSAD) }\end{array}$} \\
\hline \multicolumn{10}{|l|}{ 4. Programa Restaurantes Populares } \\
\hline \multicolumn{10}{|l|}{ 5. Programa Bancos de Alimentos } \\
\hline \multicolumn{10}{|l|}{ 6. Programa de Cozinhas Comunitárias } \\
\hline \multicolumn{10}{|l|}{ 7. Mercados Populares } \\
\hline \multicolumn{10}{|l|}{ 8. Feiras livres (Registrar os dias da semana) } \\
\hline \multicolumn{10}{|l|}{ 9. Programa de Agricultura Urbana e Periurbana } \\
\hline \multicolumn{10}{|l|}{$\begin{array}{l}\text { 10. Sistema Nacional de Segurança Alimentar e } \\
\text { Nutricional (Sisan) }\end{array}$} \\
\hline \multicolumn{10}{|l|}{ 11. Programa de Alimentação do Trabalhador } \\
\hline $\begin{array}{l}\text { 12. Programa Nacional de Alimentação do Escolar } \\
\text { (PNAE) }\end{array}$ & & & & & & & & & \\
\hline
\end{tabular}

${ }^{4}$ Se nunca existiu, registrar se tem conhecimento desse Programa.

${ }^{5}$ Considerando uma escala de 0 a 10 , onde 0 significa "péssimo" e 10 significa “ótimo", como o(a) Sr.(a) avalia o funcionamento desse programa aqui no município? Por quê? 
Capacidade instalada e SISAN em Sergipe. Silva, Fagundes e Silva

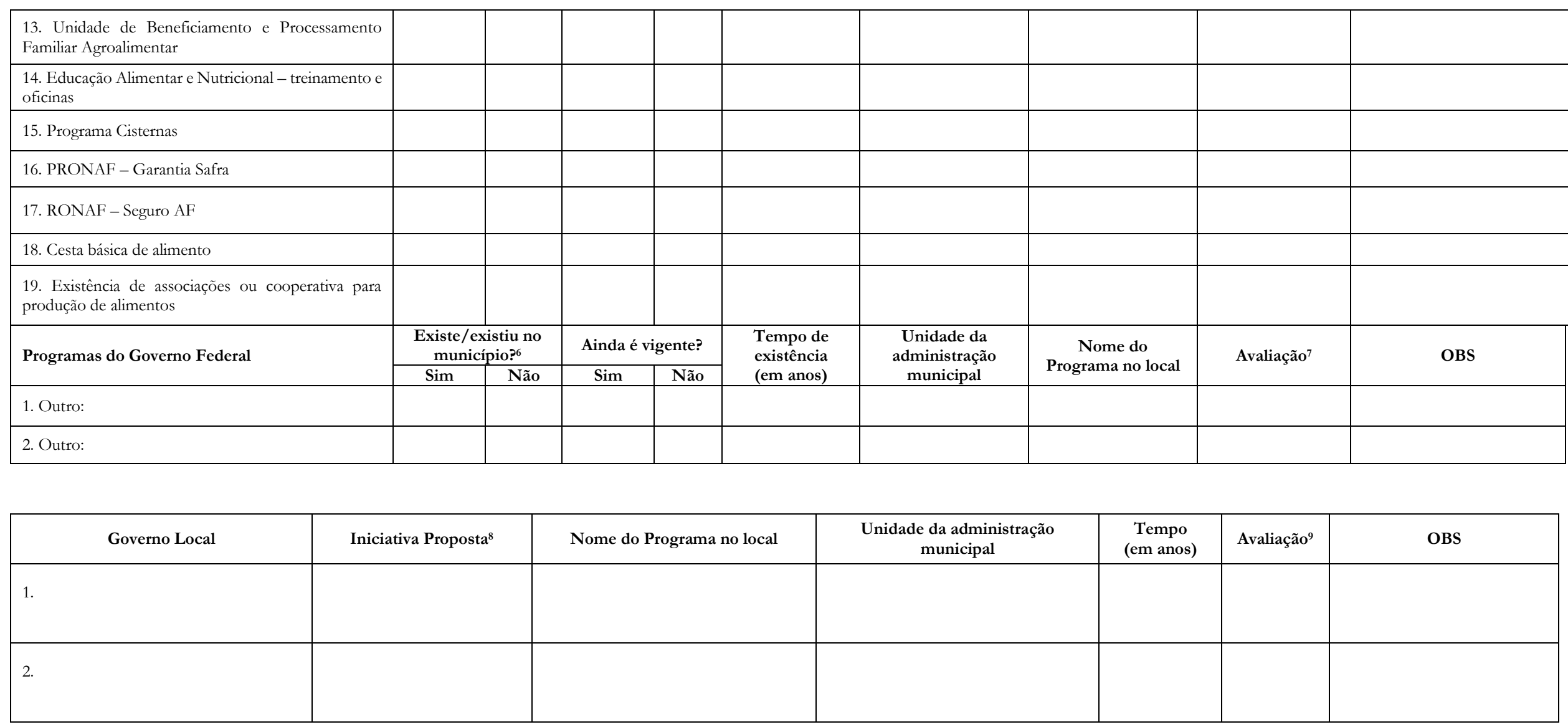

${ }^{6}$ Se nunca existiu, registrar se tem conhecimento desse Programa.

${ }^{7}$ Considerando uma escala de 0 a 10 , onde 0 significa "péssimo" e 10 significa "ótimo", como o(a) Sr.(a) avalia o funcionamento desse programa aqui no município? Por quê?

${ }^{8}$ Código Iniciativa da Proposta: $1=\$$ do Município; 2 = \$ do Estado; $3=\$$ da União

${ }^{9}$ Considerando uma escala de 0 a 10 , onde 0 significa "péssimo" e 10 significa "ótimo", como o(a) Sr.(a) avalia o funcionamento desse programa aqui no município? 
27. Existe Conselho de Segurança Alimentar e Nutricional no município (Conseam)?

( ) Sim ( ) Não

28. Se sim, como é o funcionamento do Conseam?

29. Existe o Sistema de Segurança Alimentar e Nutricional (SISAN)? ( ) Sim ～（ ） Não

30. Existe a Câmara Intersetorial de Segurança Alimentar e Nutricional (CAISAN)? ( ) Sim ～（）Não

31. O município já realizou alguma Conferência de Segurança Alimentar e Nutricional? （） Sim （ ） Não (se não, pule para 35)

32. Quantas Conferências foram realizadas no município até o momento:

33. Quais foram os produtos gerados pela Conferência? (marcar os produtos, independente do número de Conferência)

$\begin{array}{lll}(j) \text { ato legal de governo ou outro } & \left(\begin{array}{l}\text { ) documento base } \\ (\end{array}\right) \text { moção }\end{array}$

( ) relatório $\quad$ ( ) carta final do evento ( ) outros- especificar:

34. Em sua opinião qual foi o principal produto da Conferência?

35. Já realizou Reunião Territorial ou Reunião Ampliada para as Conferências de Segurança Alimentar e Nutricional? ( ) Sim

36. Gerou algum produto? (como exemplo os da questão 33) ( Sim. Quais?

38. O município tem Povos e Comunidades Tradicionais (PCT ${ }^{10}$ no território? ( ) Sim （ ) Não Quais?

39. Se sim, existem ações de SAN específicas para essa população? ( ) Sim （ ) Não Quais?

\footnotetext{
${ }^{10}$ Indígenas, Quilombolas, Matriz Africana, Povos de Terreiros, Ribeirinhos, Pescadores, Catadores e outros que o gestor considerar. Neste último, especificar.
} 